\title{
A sub-band based reconstructor for M-channel time-interleaved ADCs with missing samples
}

\author{
Anu Kalidas Muralidharan Pillai and Håkan Johansson
}

\section{Linköping University Post Print}

\section{Tweet}

N.B.: When citing this work, cite the original article.

(C2014 IEEE. Personal use of this material is permitted. However, permission to reprint/republish this material for advertising or promotional purposes or for creating new collective works for resale or redistribution to servers or lists, or to reuse any copyrighted component of this work in other works must be obtained from the IEEE.

Anu Kalidas Muralidharan Pillai and Håkan Johansson, A sub-band based reconstructor for Mchannel time-interleaved ADCs with missing samples, 2014, IEEE International Conference on Acoustics, Speech, and Signal Processing (ICASSP 2014), Florence, Italy, May 4-9.

http://dx.doi.org/10.1109/ICASSP.2014.6854378

Postprint available at: Linköping University Electronic Press http://urn.kb.se/resolve?urn=urn:nbn:se:liu:diva-103931 


\title{
A SUB-BAND BASED RECONSTRUCTOR FOR $M$-CHANNEL TIME-INTERLEAVED ADCS WITH MISSING SAMPLES
}

\author{
Anu Kalidas Muralidharan Pillai and Håkan Johansson \\ Division of Electronics Systems, Department of Electrical Engineering \\ Linköping University, SE-581 83, Sweden
}

\begin{abstract}
This paper proposes a scheme for the recovery of a uniformly sampled sequence from the output of a time-interleaved analog-to-digital converter (TI-ADC) with static time-skew errors and missing samples. Nonuniform sampling occurs due to timing mismatches between the individual channel ADCs and also due to missing input samples as some of the sampling instants are reserved for estimating the mismatches in the TI-ADC. In addition to using a non-recursive structure, the proposed reconstruction scheme supports online reconfigurability and reduces the computational complexity of the reconstructor as compared to a previous solution.
\end{abstract}

Index Terms - Nonuniform sampling, time-interleaved ADC, reconstruction, timing mismatch, FIR

\section{INTRODUCTION}

Time-interleaved analog-to-digital converters (TI-ADCs) support high sampling rates by interleaving the outputs from multiple parallel channel ADCs [1]. The individual channel ADCs in an $M$-channel TI-ADC operate at a rate that is $M$ times lower than the TI-ADC output rate. However, mismatches between the channel ADCs degrade the achievable SNDR at the output of the TI-ADC.

Several papers have addressed the problem of utilizing digital reconstructors at the output of the TI-ADC, to correct the errors introduced due to the mismatches [2-5]. However, the digital reconstructors require an estimator to estimate the various mismatch parameters like gain, offset, and timing mismatches. Estimators can be classified as either foreground or background estimators. While foreground estimators interrupt the normal operation of the TI-ADC, they achieve better convergence compared to background estimators which rely on blind techniques for estimating the mismatch, and hence, do not interrupt the normal operation of the TI-ADC.

Recently, an iterative online calibration scheme was proposed in [6] where the estimation was performed by injecting a known calibration signal to the TI-ADC input at predefined sampling instants. The samples at the output of the TI-ADC which correspond to the calibration signal are utilized to estimate the mismatch parameters. The sampling instants, where the calibration signal is inserted, are selected such that the input to the estimator contains output samples from all the channel ADCs. Since some of the sampling instants are reserved for the calibration signal, the TI-ADC input is not sampled at certain sampling instants. In order to recover the missing input samples as well as compensate for the time-skew error between the channel ADCs, an iterative reconstruction scheme using a recursive structure was proposed in [6]

In this paper, we propose a reconfigurable reconstruction scheme that corrects timing mismatches in the presence of missing samples without using recursive structures. This paper focuses on correction of static timing mismatches which is appropriate for TI-ADCs with moderate sampling frequencies and/or resolutions. Also, for the proposed reconstructor, the computational complexity which is measured in terms of the number of multiplications required to correct an output sample, is substantially lower than that of the reconstructor in [6]. Immediately following this introduction, in Section 2, we briefly review the basics of reconstruction in the presence of missing samples. In Section 3, we introduce the proposed reconstructor and illustrate the savings obtained with the help of a design example in Section 4. Section 5 concludes the paper.

\section{PREREQUISITES}

Uniform sampling of a continuous-time signal, $x_{a}(t)$, results in an output sequence $x(n)=x_{a}(n T)$, where $T$ is the sampling period. In order to ensure that the output of a TI-ADC is the uniformly sampled version of the input signal, the time-skews between the sampling clocks of the channel ADCs should be uniform. Due to nonuniform time skews, the output of the TI-ADC, $v(n)$, will be a nonuniformly sampled version of the input such that

$$
v(n)=x_{a}\left(n T+\varepsilon_{n} T\right)
$$

where $\varepsilon_{n} T$ represents, for the $n$th sample, the deviation of the actual sampling instant from the uniform sampling instant $n T$. Throughout this paper, we assume that $T=1$ for simplicity. In an $M$-channel TI-ADC, it can be assumed that the chan- 


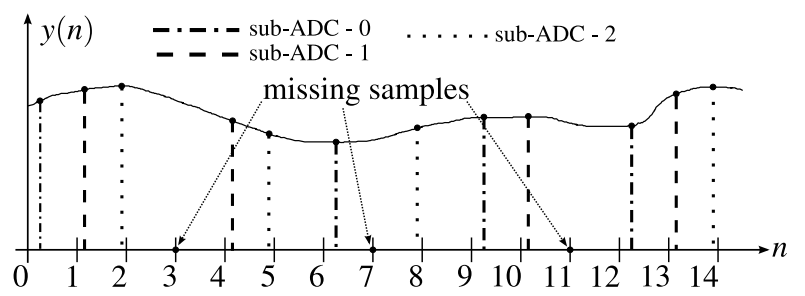

Fig. 1. Nonuniformly sampled sequence at the output of a three-channel TI-ADC with missing samples.

nel time-skew errors remain the same for a set of samples. This implies that the time-skew errors are $M$-periodic such that $\varepsilon_{n}=\varepsilon_{n+M}$ resulting in an $M$-periodic nonuniformly sampled sequence at the output of the TI-ADC.

In practice, TI-ADCs employ either foreground or background calibration techniques to estimate the time-skew errors. The estimates are then used to reconstruct the uniformly sampled sequence from the nonuniformly sampled sequence at the output of the TI-ADC. An iterative online calibration scheme was proposed in [6], where the estimation was performed by injecting a known calibration signal $c_{a}(t)$ to the TI-ADC input at predefined sampling instants $t=r M_{c}-1$ where $r$ takes on integer values. Thus, every $M_{c}$ th output sample from the TI-ADC corresponds to a nonuniformly sampled version of $c_{a}(t)$. The nonuniformly sampled calibration sequence, $c(r)$, is fed to an estimator which compares $c(r)$ with a known reference sequence, $c_{r e f}(r)=c_{a}\left(r M_{c}-1\right)$, and thereby estimates the mismatch between the channel ADCs. To ensure that $c(r)$ is composed of samples from all the channel ADCs, for an $M$-channel TI-ADC, $M_{c}$ is chosen such that $M_{c}$ and $M$ are co-prime. Unlike blind estimation techniques which suffer from slow convergence rates, the estimation technique in [6] provides faster convergence rate. However, as some of the sampling instants are reserved for the calibration signal, the input to the reconstructor $y(n)$ is given by

$$
y(n)= \begin{cases}0, & n=r M_{c}-1 \\ v(n), & \text { otherwise }\end{cases}
$$

Figure 1 illustrates the output $y(n)$ in a three-channel TI-ADC $(M=3)$ when every fourth sampling instant $\left(M_{c}=4\right)$ is used to sample the calibration signal. Hence, in addition to correcting the time-skew errors, the reconstructor used in such TI-ADCs should also recover the missing samples. Due to this, the complexity of the reconstructor will be higher than the complexity of reconstructors which correct only the timeskew errors. In [6], the reconstructor was realized using an iterative scheme with good convergence rate. However, the iterative scheme has a high computational complexity and makes use of a recursive structure which, in addition to limiting the maximum output rate [7] of the reconstructor, can also cause stability problems.

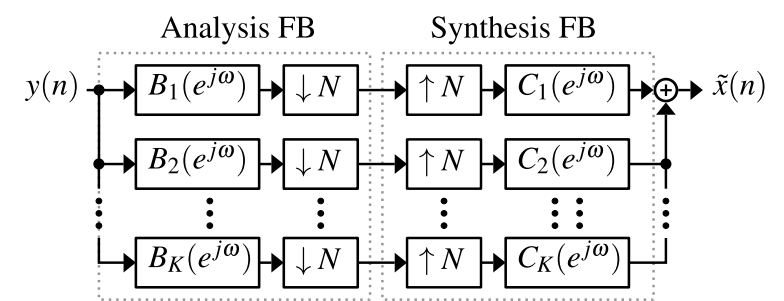

Fig. 2. Reconstruction using analysis and synthesis filters.

\section{PROPOSED RECONSTRUCTOR}

The proposed reconstructor is realized using non-recursive FIR structures, and thus, avoids the problems that can affect recursive structures. We have observed that the problem of reconstructing missing samples is similar to that of the reconstruction problem in sub-Nyquist sampled sparse multi-band signals considered in [8]. Assume that the whole Nyquist band is divided into $N$ sub-bands of equal width $\pi / N$. Then, in the case of sparse multi-band signals, only $K$ of the $N$ sub-bands are allocated to users. For such sparse multi-band signals, one of the methods to reduce the average sampling rate to the Landau minimal sampling rate is through cyclic nonuniform sampling (CNUS) [9]. In CNUS, only a subset $x\left(N n-m_{\ell}\right), \ell=1,2, \ldots, K, m_{\ell} \in[0,1, \ldots, N-1]$, of the uniform samples $x(n)$ are used. A reconstructor is then used to recover the uniformly sampled sequence $x(n)$ from the subNyquist sampled sequence $x\left(N n-m_{\ell}\right)$. A practical implementation of the CNUS is an $N$-channel TI-ADC where only $K$ of the $N$ channels are active. In [8], the reconstruction was performed using a set of analysis and synthesis filter banks (FBs) as shown in Fig. 2. Each analysis filter $B_{k}\left(e^{j \omega}\right)$, whose $N-K$ polyphase branches are equal to zero and which corresponds to the missing samples, extracts the signal from a unique active sub-band. The extracted bandlimited signal is then placed at the original active sub-band location at the output sampling rate via the downsampler, upsampler, and bandpass filter $C_{k}\left(e^{j \omega}\right)$. In the wideband TI-ADC reconstruction problem considered here, the active sub-bands are contiguous band locations starting from DC up to the maximum bandwidth of the TI-ADC, $\omega_{0}$, which is typically $80-90 \%$ of the Nyquist band.

In the proposed reconstructor, each analysis filter $B_{k}\left(e^{j \omega}\right)$ is designed such that, in addition to extracting the signal corresponding to the appropriate sub-band, each $B_{k}\left(e^{j \omega}\right)$ also makes the extracted signal uniformly spaced by compensating for the channel time-skew errors. As can be seen from (2), the samples in $y(n)$ are $M M_{c}$-periodically nonuniformly sampled version of the input $x_{a}(t)$. Hence, the proposed reconstructor will require $K=N-M$ analysis and synthesis filters where $N=M M_{c}$. 


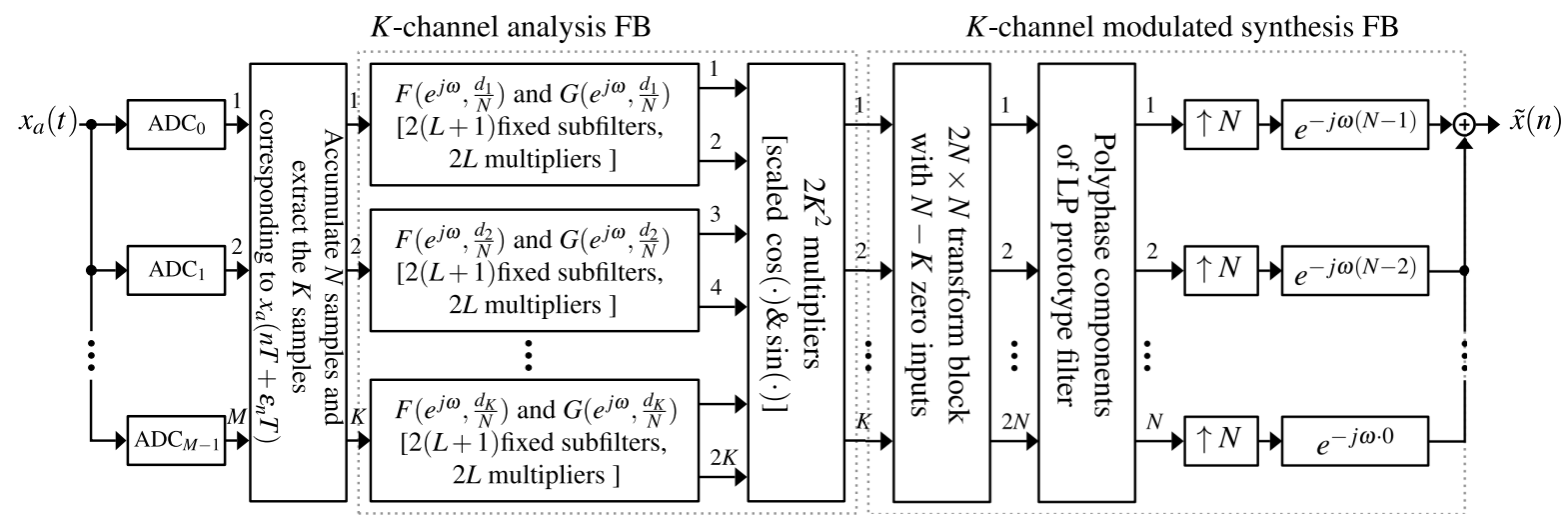

Fig. 3. Proposed reconfigurable reconstruction scheme.

\subsection{Analysis Filters}

The proposed reconstruction scheme is shown in Fig. 3. The main difference between the reconstruction problem in TIADCs and the sub-Nyquist sampling case in [8] is that, in TIADCs, the sampling instants are non-integer multiples of $T$ while [8], which considered the main principle of reconstructing sub-Nyquist sampled signals, assumed that the sampling instants are integer multiples of $T$. This implies that, here we cannot directly utilize the polyphase components of the analysis filters $B_{k}\left(e^{j \omega}\right)$. Instead, an extension of the polyphase representation is used to express the frequency response of the analysis filters $B_{k}\left(e^{j \omega}\right)$ as

$$
B_{k}\left(e^{j \omega}\right)=\sum_{\ell=1}^{K} e^{-j \omega\left(m_{\ell}+\varepsilon_{\ell}\right)} B_{k m_{\ell}}\left(e^{j \omega N}\right), \omega \in[-\pi, \pi]
$$

where $m_{\ell} \in[0,1, \ldots, N-1], \ell=1,2, \ldots, K$, are the $K$ input sampling instants, and $\varepsilon_{\ell}$ is the time-skew error corresponding to the sampling instant $m_{\ell}$. Further, while the R.H.S. of (3) is not $2 \pi$-periodic, $B_{k}\left(e^{j \omega}\right)$ is defined to be $2 \pi$-periodic. In a full-length paper under way [10], we show that $B_{k m_{\ell}}\left(e^{j \omega}\right)$ are generalized fractional-delay filters so that

$$
B_{k m_{\ell}}\left(e^{j \omega}\right) \approx \beta_{k m_{\ell}} e^{j\left(\omega\left(m_{\ell}+\varepsilon_{\ell}\right) / N+\alpha_{k m_{\ell}} \operatorname{sgn}(\omega)\right)}, \omega \in[-\pi, \pi] .
$$

In (4), $\beta_{k m_{\ell}}$ and $\alpha_{k m_{\ell}}$ are the modulus and angle, respectively, of a corresponding complex constant $c_{k m_{\ell}}$. It is shown in [10] that the vector $\mathbf{c}_{k}$, containing all the $K$ complex constants $c_{k m_{\ell}}$, $m_{\ell} \in[0,1, \ldots, N-1], \ell=1,2, \ldots, K$, can be determined using matrix inversion as

$$
\mathbf{c}_{k}=\mathbf{A}^{-1} \mathbf{b}_{k} \text {. }
$$

Here, $\mathbf{A}$ is a $K \times K$ matrix with elements $a_{n_{\ell} m_{\ell}}=e^{j 2 \pi n_{\ell}\left(m_{\ell}+\varepsilon_{\ell}\right) / N}$, $n_{\ell} \in[0, \pm 1, \ldots, N / 2]$ if $N$ is even or $n_{\ell} \in[0, \pm 1, \ldots, \pm(N-$ $1) / 2]$ if $N$ is odd, $m_{\ell} \in[0,1, \ldots, N-1], \ell=1,2, \ldots, K$, determined by the $K$ sampling points $m_{\ell}$ and the $K$ active bands $n_{\ell}$. Further, one of the elements of $\mathbf{b}_{k}$ is unity, its position being determined by the active band $n_{\ell}$. The remaining elements in $\mathbf{b}_{k}$ are zero.

\subsubsection{Reconfigurability}

In order to make the reconstructor online reconfigurable without any redesign, the polyphase branches of $B_{k}\left(e^{j \omega}\right)$ are implemented using the structure in [11] so that $B_{k m_{\ell}}\left(e^{j \omega}\right)$ in (4) are expressed as

$$
B_{k m_{\ell}}\left(e^{j \omega}\right)=\gamma_{k m_{\ell}} F\left(e^{j \omega}, d_{\ell} / N\right)+\zeta_{k m_{\ell}} G\left(e^{j \omega}, d_{\ell} / N\right)
$$

where $\gamma_{k m_{\ell}}=\beta_{k m_{\ell}} \cos \left(\theta_{k m_{\ell}}\right), \zeta_{k m_{\ell}}=\beta_{k m_{\ell}} \sin \left(\theta_{k m_{\ell}}\right), \theta_{k m_{\ell}}=$ $\alpha_{k m_{\ell}}+\pi / 4^{1}$, and $d_{\ell}=m_{\ell}+\varepsilon_{\ell}$. As shown in [11], $F\left(e^{j \omega}, d_{\ell} / N\right)$ and $G\left(e^{j \omega}, d_{\ell} / N\right)$ can be realized in terms of a polynomial FIR structure with $L+1$ fixed subfilters $F_{q}(z)$ and $G_{q}(z)$, respectively, each weighted with $\left(d_{\ell} / N\right)^{q}, q=0,1, \ldots, L$. Thus, all the $B_{k m_{\ell}}\left(e^{j \omega}\right)$ are expressed using a common set of fixed subfilters $F_{q}\left(e^{j \omega}\right)$ and $G_{q}\left(e^{j \omega}\right)$ since (4) can be rewritten as

$B_{k m_{\ell}}\left(e^{j \omega}\right)=\gamma_{k m_{\ell}} \sum_{q=0}^{L}\left(\frac{d_{\ell}}{N}\right)^{q} F_{q}\left(e^{j \omega}\right)+\zeta_{k m_{\ell}} \sum_{q=0}^{L}\left(\frac{d_{\ell}}{N}\right)^{q} G_{q}\left(e^{j \omega}\right)$.

The different polyphase branches can be obtained via different sets of values for $\gamma_{k m_{\ell}}, \zeta_{k m_{\ell}}$, and $d_{\ell}$. Also, when the timeskew errors change, only the general multipliers corresponding to $\gamma_{k m_{\ell}}, \zeta_{k m_{\ell}}$, and $d_{\ell}$ need to be updated with the corresponding new values.

\subsection{Synthesis Filters}

As in [8], the fixed bandpass synthesis filters $C_{k}\left(e^{j \omega}\right)$ can be efficiently realized using a cosine modulated FB. The prototype filter is a lowpass filter with cutoff frequency at $\pi / 2 N$ [12]. Thus, the overall complexity of the synthesis FB correspond to that of the prototype filter plus the cost of a real or complex transform block. A fast-transform algorithm can be used to make the cost of such a transform block low compared to the cost of the filters.

\footnotetext{
${ }^{1}$ The additional phase shift of $\pi / 4$ is required to match the analysis and synthesis FBs since the subbands in the passband region overlap and because we use cosine modulated FBs. It is similar to using additional phase constants that are used for matching in conventional cosine modulated FBs [12].
} 


\subsection{Reconstructor Design}

The Fourier transform (FT) of the reconstructed output $\tilde{x}(n)$ can be expressed in terms of the FT of $x(n)$, i.e., $X\left(e^{j \omega}\right)$, as

$$
\tilde{X}\left(e^{j \omega}\right)=V_{0}\left(e^{j \omega}\right) X\left(e^{j \omega}\right)+\sum_{p=1}^{N-1} V_{p}\left(e^{j \omega}\right) X\left(e^{j(\omega-2 \pi p / N)}\right)
$$

where $V_{0}\left(e^{j \omega}\right)$ is the distortion function and $V_{p}\left(e^{j \omega}\right), p=$ $1,2, \ldots, N-1$ are the aliasing functions with

$$
V_{p}\left(e^{j \omega}\right)=\frac{1}{N} \sum_{k=1}^{K} B_{k}\left(e^{j(\omega-2 \pi p / N)}\right) C_{k}\left(e^{j \omega}\right)
$$

for $p=0,1, \ldots, N-1$. To have near perfect reconstruction, the distortion term and the aliasing terms in (8) should approximate unity and zero, respectively, in the passband region $\omega \in\left[-\omega_{0}, \omega_{0}\right]$. Since the $2(L+1)$ subfilters in (7) are fixed, whenever the time-skew errors change, it suffices to redetermine the complex coefficients $\mathbf{c}_{k}$ which, as in (5), can be done using matrix inversion. The coefficients of the $2(L+1)$ fixed subfilters, $F_{q}\left(e^{j \omega}\right)$ and $G_{q}\left(e^{j \omega}\right)$, are determined offline such that the distortion and aliasing functions approximate unity and zero, respectively, in the passband $\omega \in\left[-\omega_{0}, \omega_{0}\right]$ with certain tolerances $\delta_{0}$ and $\delta_{1}$ according to

$$
\begin{gathered}
\left|V_{0}\left(e^{j \omega}\right)-1\right| \leq \delta_{0}, \omega \in\left[-\omega_{0}, \omega_{0}\right], \\
\left|V_{p}\left(e^{j \omega}\right)\right| \leq \delta_{1}, \omega \in \Omega_{p}
\end{gathered}
$$

for $p=1,2, \ldots, N-1$. Here, $\Omega_{p}$ represents the shifted versions of the passband that fall into the band $[-\pi, \pi]$.

\section{DESIGN EXAMPLE}

In this section, in order to illustrate the savings obtained by using the proposed reconstructor, we use the four-channel TIADC $(M=4)$ case considered in Example A in Section VI of [6]. Hence, it is assumed that the timing mismatches in the channel ADCs are $\varepsilon_{0}=0.01, \varepsilon_{1}=-0.05, \varepsilon_{2}=0.04$, and $\varepsilon_{3}=-0.03$, and the bandwidth of the reconstructor, $\omega_{0}$, is $0.8 \pi$. Also, as in [6], we assume that every seventh sample is used by the estimator, i.e., $M_{c}=7$. Thus, for the proposed reconstructor, $N=28, K=24$, and the input sampling instants are $m_{\ell}=\{[0: 5],[7: 12],[14: 19],[21: 26]\}$.

The reconstructor was designed such that, after reconstruction, the aliasing terms are below $-50 \mathrm{~dB}$ which corresponds to three iterations of the reconstructor in [6] as can be seen from Fig. 9(c) in [6]. At first, the prototype filter for the fixed synthesis FB was designed to be a power-symmetric lowpass filter of order 400 and cutoff frequency at $0.6 \pi / 56$. To keep the aliasing terms below $-50 \mathrm{~dB}$, the reconfigurable analysis filters require 10 subfilters $(L=4), F_{q}(z)$ and $G_{q}(z)$, each of order 20. Thus, for the proposed method, the overall order of the reconstructor is 960 . However, the computational complexity measured in terms of the number of multiplications per output sample is only around 75 assuming a
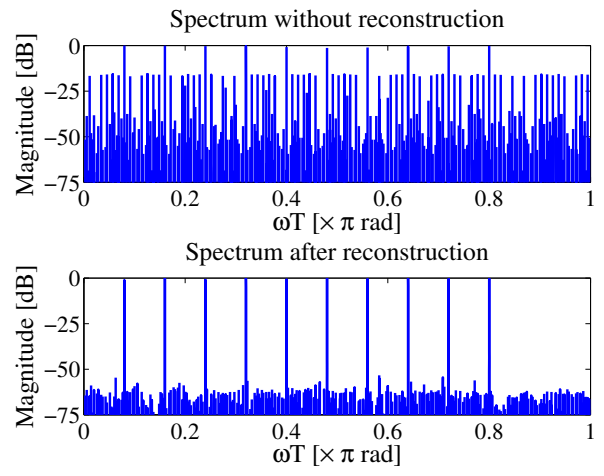

Fig. 4. Spectrum before and after reconstruction using the reconstructor in the design example.

straight-forward implementation of the transform block. On the other hand, the iterative reconstructor in [6] would require a reconstructor of order 396. The structure in [6] requires 36 general multipliers operating at the input/output rate while the proposed structure requires 48 general multipliers at the input/output rate. In [6], for every block of seven output samples, the VDF subfilters operate only on six samples while a highpass filter is required for one of the seven samples. Hence, the average computation complexity is around 550 multiplications per output sample. It can be seen that, while the overall order of the proposed reconstructor is higher compared to the structure in [6], it requires significantly fewer multiplications per corrected output sample. Also, due to its non-recursive structure, the proposed method is free from stability issues that can affect the iterative reconstructor in [6]. Also, in [6], the use of recursive structure limits the maximum rate at which the reconstructor can operate [7]. Like in [6], the performance of the reconstructor is evaluated by passing a multi-tone sinusoidal input $x(n)=\sum_{r=1}^{10} \sin \left(n \omega_{r}\right)$, where $\omega_{r}=2 \pi r / 25$. The spectrum of the nonuniformly sampled TI-ADC output with missing samples, $y(n)$, and that of the reconstructed sequence, $\tilde{x}(n)$ is shown in Fig. 4.

\section{CONCLUSION}

This paper proposed a reconstruction scheme for the recovery of a uniformly sampled sequence from the output of a TI-ADC with static time-skew errors and missing samples. In order to reduce the computational complexity, the whole Nyquist band was divided into a number of subbands due to which we could describe the reconstruction in terms of analysis and synthesis FBs. Also, for reconfigurability, polynomial FIR filters with fixed subfilters were used to realize the analysis FB. With the help of a design example, it was shown that the proposed reconstructor gives significant savings in computational complexity. 


\section{REFERENCES}

[1] W. C. Black and D. A. Hodges, "Time interleaved converter arrays," IEEE J. Solid-State Circuits, vol. 15, no. 6, pp. 1022-1029, Dec. 1980.

[2] J. Selva, "Functionally weighted lagrange interpolation of band-limited signals from nonuniform samples," IEEE Trans. Signal Process., vol. 57, no. 1, pp. 168181, Jan. 2009.

[3] S. Tertinek and C. Vogel, "Reconstruction of nonuniformly sampled bandlimited signals using a differentiator-multiplier cascade," IEEE Trans. Circuits Syst. I, vol. 55, no. 8, pp. 2273-2286, Sep. 2008.

[4] H. Johansson and P. Löwenborg, "Reconstruction of nonuniformly sampled bandlimited signals by means of time-varying discrete-time FIR filters," EURASIP J. Advances Signal Process., vol. 2006, Jan. 2006.

[5] T. Strohmer and J. Tanner, "Fast reconstruction algorithms for periodic nonuniform sampling with applications to time-interleaved ADCs," in Proc. IEEE Int. Conf. Acoustics, Speech Signal Process., vol. 3, 2007.

[6] K. M. Tsui and S. C. Chan, "A novel iterative structure for online calibration of $M$-channel time-interleaved ADCs," IEEE Trans. Instrum. Meas., vol. 63, no. 2, pp. 312-325, Feb. 2014.

[7] M. Renfors and Y. Neuvo, "The maximum sampling rate of digital filters under hardware speed constraints," IEEE Trans. Circuits Syst., vol. 28, no. 3, pp. 196-202, Mar. 1981.

[8] A. K. M. Pillai and H. Johansson, "Efficient reconfigurable scheme for the recovery of sub-Nyquist sampled sparse multi-band signals," in Proc. IEEE Global Conf. Signal Information Process., Dec. 2013.

[9] M. Mishali and Y. Eldar, "Sub-Nyquist sampling," IEEE Signal Process. Mag., vol. 28, no. 6, pp. 98-124, Nov. 2011.

[10] A. K. M. Pillai and H. Johansson, "Efficient recovery of sub-Nyquist CNUS sparse multi-band signals using analysis and modulated synthesis filter banks," in preparation.

[11] H. Johansson and A. Eghbali, "FIR filter with variable fractional delay and phase shift: Efficient realization and design using reweighted $\ell_{1}$-norm minimization," in Proc. IEEE Int. Symp. Circuits Syst., Beijing, China, May 19-23 2013.

[12] P. P. Vaidyanathan, Multirate Systems and Filter Banks. Prentice-Hall, Englewood Cliffs, NJ, USA, 1993. 